where the personal contact with the GP often gave valuable insights that could not easily be conveyed in a formal referral letter. This focus on individual patients led to the raising of broader issues and more general applications of points raised. As the meetings went on, a freer exchange of information evolved, with a psychiatric input being available for those patients whom the GP saw but did not consider for formal referral. Where the GP is managing a patient with psychological difficulties, we have found that discussion and elaboration of issues often serves to strengthen the GP's role in treatment. It was not the aim of these sessions to dissuade formal referral but it has become apparent that there has been a decrease in such referrals, with 12 patients being referred to the CMHT over the six months of these meetings compared with 26 in the corresponding six month period of the previous year (total number referred in previous year $=48$ ). There has been no other change apparent to account for the fall-off and it appears reasonable to attribute this to the regular liaison meeting.

It is possible that where the GP is unsure of the management of particular patients, then a forum for discussion allows clarification of issues and it has been apparent that the GPs often prefer to continue their management of patients where possible while the security of knowing that review is possible at later meetings has enabled this to occur. It does appear that the investment of time while being a beneficial experience over the long term for both GPs and psychiatrist also has more immediate benefits.

UMDS, Guys \& St Thomas' Hospitals

London SEI

H. DOYLE

\section{Working with clozapine}

\section{DeAr Sirs}

Dr A. Mahmood described a 19-year-old schizophrenic patient who developed chickenpox while on clozapine (Psychiatric Bulletin, 1991, 15, 702). He stated that the patient's WBC and neutrophils dropped sharply. However, I wish to point out that the patient did not become neutropenic as the lowest recorded neutrophil count and WBC were $3.31 \times 10^{9}$ per litre and $4.66 \times 10^{9}$ per litre respectively during the episode. Hence the patient could be recommenced on clozapine.

SAMIR N. SHAH

Sandoz Pharmaceuticals

Clozaril Patient Monitoring Service

Frimley Business Park

Frimley, Camberley, Surrey GU16 5SG

\section{Working with clozapine - it can be done}

The clinical hazards experienced while working with clozapine, recounted by Adams \& Essali (Bulletin
$1991,15,336-338$ ), may have partly reflected the constraints of the drug trial into which the patients discussed had been recruited. In this open study, administration of clozapine followed a fixed-dose schedule rather than clinical titration as is generally the rule with antipsychotic drugs. This may have led to the use of higher doses than would have been reached in routine clinical management and thus an increased risk of adverse side effects. Further, the sample of refractory schizophrenic patients featured in the study included a number for whom it proved impossible to withdraw their previous antipsychotic medication. In such cases, clozapine was tested as an adjunctive rather than single treatment, as Adams \& Essali mention. Whether using clozapine in combination with other antipsychotic drugs compromises its therapeutic efficacy or increases the risk of side effects remains unclear.

The clinical problems described by Adams \& Essali are undeniably part of the risk-benefit balance which needs to be considered by a clinician starting patients on clozapine. Further, the arrangements for haematological monitoring and prescription of the drug may be time-consuming. Nevertheless, it would be a shame if, for these reasons, clinicians shied away from the use of the drug in those for whom it might offer clear therapeutic benefit.

M. BRistow SteVEn HiRsch

Charing Cross Hospital Medical School T. R. E. BARNES

Fulham Palace Road

London W6 8RF

\section{Psychiatry of opera}

DEAR Sirs

Dr Jones' six-part series on the psychiatry of opera is easy prey for hostile criticism. I had hoped that others would reply to it, but in their absence it is necessary to point out, in the interests of scholarship, some of the grave deficiencies of Dr Jones' writings. One critic expressed his view after the first instalment, but Dr Jones admonished him for being too impatient (Psychiatric Bulletin, 1990, 14, 563-564). It is appropriate now to comment on the entire series.

Dr Jones correctly noted that limitations of space could not do justice to his subject but he failed to use whatever space he had. First, he unnecessarily described the lives of the composers and offered us the plots of the operas. This kind of background information is readily found in most encyclopedias, opera programmes or Kobbé's Complete Opera Book (Harewood, 1976) which he should have cited. In 1991, the bicentenary of the death of Mozart, the casual reader has been engulfed with numerous versions of his biography without an additional contribution in the present opera series. 